\title{
A comparison of the fixed combination of latanoprost and timolol with the unfixed combination of brimonidine and timolol in patients with elevated intraocular pressure. A six month, evaluator masked, multicentre study in Europe
}

\author{
J García-Sánchez, J-F Rouland, D Spiegel, B Pajic, I Cunliffe, C Traverso, J Landry
}

Br J Ophthalmol 2004;88:877-883. doi: 10.1136/bjo.2003.029330

\begin{abstract}
Purpose: To compare the intraocular pressure (IOP) reducing effect and safety of fixed combination (FC) latanoprost/ timolol with unfixed combination (UFC) brimonidine/timolol in patients with increased IOP.

Methods: In this 6 month, randomised, evaluator masked, parallel group European study, patients with glaucoma or ocular hypertension and IOP $\geqslant 21 \mathrm{~mm} \mathrm{Hg}$ on monotherapy or $>16 \mathrm{~mm} \mathrm{Hg}$ on dual therapy received either FC latanoprost/timolol at 8:00AM or UFC brimonidine/timolol at $8: 00 \mathrm{AM}$ and $8: 00 \mathrm{PM}$. The primary outcome was the difference from baseline to month 6 in mean diurnal IOP reduction.

Results: 325 of 334 randomised patients were included in intent to treat analyses (FC latanoprost/timolol, 163; UFC brimonidine/timolol, 162). Baseline diurnal IOP levels were similar: FC latanoprost/timolol, 26.4 (SD 2.7) mm Hg; UFC brimonidine/timolol, 26.5 (SD 2.8) $\mathrm{mm} \mathrm{Hg}(p=0.851)$. At month 6, levels were 16.9 (SD 2.8) $\mathrm{mm} \mathrm{Hg}$ in FC latanoprost/timolol patients and 18.2 (SD 3.1 ) $\mathrm{mm} \mathrm{Hg}$ in UFC brimonidine/timolol patients $(p<0.001)$. No adverse events were reported by $76.4 \%$ and $75.5 \%$ of patients receiving FC latanoprost/timolol versus UFC brimonidine/ timolol, respectively. Larger proportions of brimonidine/ timolol treated patients reported study medication related adverse events $(18.6 \% \vee 7.3 \%)$ and discontinued study participation because of this $(10.8 \% \vee 1.8 \%)$.

Conclusion: Fixed combination latanoprost/timolol administered once daily is both more effective and better tolerated than twice daily dosing with UFC brimonidine/timolol.
\end{abstract}

$\mathrm{T}$ pical hypotensive medication is considered the treatment of choice in the initial management of increased intraocular pressure (IOP) in patients with glaucoma. ${ }^{12}$ Target IOP levels are not always achieved with the use of one agent, however, and many patients require combination therapy. Several new and effective IOP lowering drugs have additive effects when used in combination with the $\beta$ adrenergic receptor antagonist timolol. . $^{3-6}$

Latanoprost, the only prostaglandin analogue indicated for first line use as an ocular hypotensive in Europe and the United States, lowers IOP levels by increasing uveoscleral outflow with little or no effect on aqueous humour production, ${ }^{78}$ while $\beta$ blockers are believed to reduce aqueous humour formation. ${ }^{9}$ The concomitant administration of latanoprost and timolol produces an additive IOP reducing effect. ${ }^{10}{ }^{11}$ Because complex, multidrug regimens can reduce patient compliance, ${ }^{12}{ }^{13}$ a fixed formulation of latanoprost
$0.005 \%$ and timolol $0.5 \%$ has been made available. Once daily administration of this combination is well tolerated and reduces IOP more effectively than either individual component alone in patients with open angle glaucoma and ocular hypertension. ${ }^{\text {.14 } 15}$

Brimonidine, a selective $\alpha_{2}$ agonist ocular hypotensive agent, acts by reducing aqueous humour production and increasing uveoscleral outflow. ${ }^{16}{ }^{17}$ Compared with timolol in patients with open angle glaucoma or ocular hypertension, brimonidine dosed twice daily produces similar ${ }^{18} 19$ or significantly lower ${ }^{20}$ IOP levels when measured 2 hours after a morning dose. Twelve hours after the evening dose (trough), mean decreases in IOP are consistently and significantly greater in timolol treated patients, ${ }^{18-20}$ supporting the brimonidine labelling recommendation of three times daily dosing. ${ }^{21}$ This study compares the effect on IOP of the fixed combination (FC) of latanoprost $0.005 \%$ and timolol $0.5 \%$ with that of the unfixed combination (UFC) of brimonidine $0.2 \%$ and timolol $0.5 \%$ in patients with open angle glaucoma or ocular hypertension who previously were uncontrolled on monotherapy or dual therapy. Although the recommended brimonidine dosing regimen is three times daily, twice daily dosing appears to be standard practice.

\section{METHODS}

\section{Study design}

This six month, randomised, evaluator masked, multicentre study was conducted at 34 sites in France, Germany, Italy, Spain, Switzerland, and the United Kingdom. The protocol was reviewed and approved by an institutional review board or independent ethics committee at each study site. The research was conducted in accordance with the ethical standards maintained in the Declaration of Helsinki. All patients provided written consent.

\section{Patients}

Patient selection criteria are summarised in the box.

\section{Study protocol}

Patients were assessed for eligibility at a screening visit in the 4 weeks before baseline when a medical and ocular history was taken, and IOP measurement (with a calibrated Goldmann applanation tonometer), Snellen visual acuity measurement, and visual field testing using automated perimetry preferably with a Humphrey Field Analyzer (if not performed within the past year) were performed. Abnormal findings of a slit lamp examination (biomicroscopy) and ophthalmoscopy were graded as mild, moderate,

Abbreviations: FC, fixed combination; IOP, intraocular pressure; UFC, unfixed combination 


\section{Patient selection}

Inclusion criteria:

- $\geqslant 18$ years of age

- Unilateral or bilateral primary open angle, pigmentary, or exfoliative glaucoma or ocular hypertension (IOP $\geqslant 21 \mathrm{~mm} \mathrm{Hg}$ )

- At screening, inadequately responsive to monotherapy or dual therapy (IOP > $16 \mathrm{~mm} \mathrm{Hg}$ )

- At baseline, following washout of previous therapy: (1) mean 8:00AM IOP $\geqslant 25 \mathrm{~mm} \mathrm{Hg}$ and an increase in IOP $\geqslant 3 \mathrm{~mm} \mathrm{Hg}$ from screening; (2) best corrected visual acuity $\geqslant 20 / 80$; (3) able to comply with protocol requirements

Exclusion criteria (history of one or more)

- Acute angle closure glaucoma

- Closed or barely open anterior chamber angle

- Argon laser trabeculoplasty or any ocular surgery or inflammation/infection within 3 months of screening

- Ocular filtering surgery

- Other abnormal ocular conditions

- Sensitivity to benzalkonium chloride or any other component of drug solutions

- A condition in which treatment with a $\beta$ adrenergic receptor antagonist is contraindicated

- Concurrent use of monamine oxidase inhibitors or tricyclic antidepressants

- Use of an investigational medication within 1 month before screening

- Use of systemic medication known to affect IOP unless both patient and dosage were stable for preceding 3 months and no change in dosage expected during study period

- Pregnancy or lactation

or severe. For eligible patients, current ocular hypotensive treatments were suspended with required prebaseline washout periods of 4 weeks for $\beta$ adrenergic receptor antagonists and prostaglandin analogues, 2 weeks for adrenergic agonists, and 5 days for cholinergic agonists and carbonic anhydrase inhibitors. Patients previously using $\beta$ adrenergic receptor antagonists or prostaglandin analogues had a safety visit after 2 weeks of washout and, if their IOPs were considered detrimental, were excluded from the study at the discretion of the investigator.

Patients were evaluated during four study visits: at baseline and months 1, 3, and 6. Patients with ongoing adverse events at month 6 had an additional visit 2 weeks after study completion. At each study visit, IOP was measured in triplicate in each eye alternating between eyes and starting with the right eye at 8:00AM (before scheduled dosing), 12:00 noon, and 4:00PM; the mean of the three measurements was used as "the IOP" for a given time point. At each visit, lid/slit lamp examinations using biomicroscopy and Snellen visual acuity measurement tests were performed and use of concomitant medications were recorded; ophthalmoscopy was performed at months 3 and 6. Blood pressure and heart rate were recorded at all visits except at the two week IOP safety check.

At each site, eligible patients were assigned randomly (1:1) to either of the two treatment groups using randomisation envelopes (Pharmacia Corporation, Peapack, NJ, USA).
Patients and study personnel were cautioned not to reveal the medication assignment or the frequency of dosing to masked evaluators. Study medications were prepackaged in black polyprophylene containers. Patients were reminded to change study medication bottles every 4 weeks, and all medication bottles and their containers were returned at the patient's next visit. Each medication bottle contained $2.5 \mathrm{ml}$ of ophthalmic solution.

Eyes that met all inclusion and no exclusion criteria were designated as study eyes. In patients with bilateral disease with only one eligible eye, the fellow eye also could be treated with study drug if no exclusion criteria applied to that eye. Patients assigned to receive FC latanoprost/timolol were instructed to instil one drop every morning at 8:00AM, beginning on the morning after the baseline visit. Patients assigned to receive UFC brimonidine/timolol were instructed to instil one drop of each medication every morning at 8:00AM and every evening at 8:00PM, with the timolol drop instilled 5 minutes before the brimonidine drop; the first drops were to be instilled in the evening of the baseline visit. No other IOP reducing treatment was permitted, and systemic carbonic anhydrase inhibitors were prohibited. Study treatment was discontinued if either the investigator or the patient thought that it was not in the patient's best interest to continue, or if the patient became pregnant.

Ocular findings and adverse events regardless of relation to treatment were monitored throughout. Investigators recorded observed adverse events, as well as those reported spontaneously by patients and those elicited by questioning. Investigators classified adverse events as mild, moderate, or severe. Serious adverse events from which the patient had not recovered at the post study visit were followed until they resolved or were chronic or stable.

\section{Variables and analyses}

Only study eyes were included in efficacy analyses. If both eyes were study eyes, the mean value of IOP measurements across eyes was used. The primary efficacy outcome was the diurnal IOP change from the baseline measurement to month 6. Diurnal IOP was calculated as the mean of IOP measurements at 8:00AM (before dosing), 12:00 noon, and 4:00PM at each study visit. A $t$ test evaluated whether the difference in diurnal IOP change between treatment groups was significant. This analysis was supported by an analysis of covariance (ANCOVA) with baseline IOP as the covariate and country as an additional explanatory variable; a 95\% confidence interval (CI) was calculated. These procedures were applied to the secondary outcomes, change in IOP by time of day and per cent change in diurnal IOP from baseline to the month 6 visit. Additional secondary efficacy outcomes were proportions of patients reaching specific IOP levels and achieving different levels of percent IOP reduction.

Intent to treat (ITT) analyses included all randomised patients who had observed IOP values at baseline and at least at one visit after baseline. If one or two IOP values at a specific time within a visit were missing, the average of remaining measures for that time was used. A missing IOP for a specific time at months 3 or 6 was replaced with the non-missing value for that time from the closest previous visit; if IOP levels were still missing within a visit, the average of remaining IOPs was used as the diurnal IOP value for the visit. The population evaluated included only randomised patients without a major protocol violation who provided IOP measurements at the baseline and month 6 visits; no missing data were imputed.

Safety analyses included all treated eyes. Adverse events were listed by treatment assignment and by patient. Results of heart rate, blood pressure, biomicroscopy, and 


\begin{tabular}{|c|c|c|}
\hline & $\begin{array}{l}\text { Fixed combination } \\
\text { latanoprost/timolol } \\
(n=163)\end{array}$ & $\begin{array}{l}\text { Unfixed combination } \\
\text { brimonidine/timolol } \\
(n=162)\end{array}$ \\
\hline \multicolumn{3}{|l|}{ Sex, n (\%) } \\
\hline Male & $73(44.8)$ & $73(45.1)$ \\
\hline Female & $90(55.2)$ & $89(54.9)$ \\
\hline \multicolumn{3}{|l|}{ Age (years) } \\
\hline Mean & 65.5 & 65.1 \\
\hline SD & 10.30 & 11.56 \\
\hline Range & $33-91$ & $23-87$ \\
\hline \multicolumn{3}{|l|}{ Ethnic origin, $n$ (\%) } \\
\hline White & $158(96.9)$ & $160(98.8)$ \\
\hline Black & $0(0.0)$ & $1(0.6)$ \\
\hline Hispanic & $4(2.5)$ & $1(0.6)$ \\
\hline Other & $1(0.6)$ & $0(0.0)$ \\
\hline \multicolumn{3}{|l|}{ Eye colour of study eye(s), n (\%) } \\
\hline Homogeneously blue, grey or green & $48(29.4)$ & $48(29.6)$ \\
\hline Homogeneously brown & $85(52.1)$ & $88(54.3)$ \\
\hline Blue-brown/grey-brown & $19(11.7)$ & $14(8.6)$ \\
\hline Green-brown & $8(4.9)$ & $6(3.7)$ \\
\hline Yellow-brown & $3(1.8)$ & $6(3.7)$ \\
\hline \multicolumn{3}{|l|}{ Study eye(s), n (\%) } \\
\hline Right & $41(25.2)$ & $34(21.0)$ \\
\hline Leff & $33(20.2)$ & $31(19.1)$ \\
\hline Both & $89(54.6)$ & $97(59.9)$ \\
\hline \multicolumn{3}{|l|}{ Treated eye(s), n (\%) } \\
\hline Right & $27(16.6)$ & $26(16.0)$ \\
\hline Leff & $37(22.7)$ & $30(18.5)$ \\
\hline Both & $99(60.7)$ & $106(65.4)$ \\
\hline \multicolumn{3}{|l|}{ Diagnosis of study eye(s), n (\%) } \\
\hline Primary open angle glaucoma & $119(73.0)$ & $111(68.5)$ \\
\hline Ocular hypertension & $37(22.7)$ & $43(26.5)$ \\
\hline Other specifications & $4(2.5)$ & $7(4.3)$ \\
\hline Mixed* $^{*}$ & $3(1.8)$ & $1(0.6)$ \\
\hline \multicolumn{3}{|l|}{ Duration of condition (study eye) } \\
\hline$<6$ months & $11(6.7)$ & $6(3.7)$ \\
\hline 6 to $<36$ months & $51(31.3)$ & $51(31.5)$ \\
\hline 36 to $<120$ months & 78 (47.9) & 75 (46.3) \\
\hline$\geqslant 120$ months & $23(14.1)$ & $30(18.5)$ \\
\hline
\end{tabular}

ophthalmoscopy evaluations were summarised by visit and examined for within group differences at months 1,3 , and 6 .

Before study initiation, it was determined that at least 113 evaluable patients were needed per treatment to detect a difference of $1.5 \mathrm{~mm} \mathrm{Hg}$ in mean diurnal IOP reduction between treatments using a $t$ test at a significance level of 0.05 , with a power of 0.80 , and assuming a standard deviation of $4.0 \mathrm{~mm} \mathrm{Hg}$. A total of 300 patients was targeted for enrolment because of previously reported high withdrawal rates associated with brimonidine therapy. In one 12 month, multicentre trial, $47 \%$ of 292 patients receiving brimonidine therapy withdrew from the study primarily during the 3 to 6 months after enrolment, with $26 \%$ of withdrawals because of adverse events.

\section{RESULTS}

In total, 334 patients were randomised: 166 to the FC latanoprost/timolol group and 168 to the UFC brimonidine/ timolol group. Three patients in the latanoprost/timolol group and six in the brimonidine/timolol group withdrew from the study before 1 month and were excluded from ITT analyses ( $\mathrm{n}=325 ;$ 163, FC latanoprost/timolol; 162, UFC brimonidine/ timolol). Demographic and other baseline characteristics were similar between groups (table 1). No significant differences between groups in any measure of pretreatment ocular conditions and no obvious differences in the use of concomitant medications were noted.

Six per cent (10/166) of patients treated with latanoprost/ timolol versus $17.3 \%(29 / 168)$ of patients receiving brimonidine/timolol withdrew from the study (table 2). In all, 276 patients were included in the evaluable population (144, FC latanoprost/timolol; 132, UFC brimonidine/timolol); the 58 exclusions were due primarily to early discontinuations (10/ 22, FC latanoprost/timolol; 27/36, UFC brimonidine/timolol).

\section{Efficacy results}

Demographic characteristics and efficacy findings were similar in the ITT and evaluable populations; the following focuses on the ITT analyses. Baseline diurnal IOP levels were similar $(p=0.851$; table 3 ; fig 1$)$. Between baseline and month 6, mean (SD) diurnal IOP decreased 9.5 (3.4) $\mathrm{mm} \mathrm{Hg}$ in the latanoprost/timolol group and 8.2 (3.4) $\mathrm{mm} \mathrm{Hg}$ in the brimonidine/timolol group $(\mathrm{p}<0.001 ; t$ test $)$. The adjusted difference (ANCOVA) between groups was $-1.33 \mathrm{~mm} \mathrm{Hg}$

Table 2 Patient disposition

\begin{tabular}{lll}
\hline & $\begin{array}{l}\text { Fixed combination } \\
\text { latanoprost/timolol, } \\
\mathbf{n}(\%)\end{array}$ & $\begin{array}{l}\text { Unfixed combination } \\
\text { brimonidine/timolol, } \\
\mathbf{n}(\%)\end{array}$ \\
\hline Total randomised & $166(100.0)$ & $168(100.0)$ \\
Completed study & $156(94.0)$ & $139(82.7)$ \\
Withdrew from study & $10(6.0)$ & $29(17.3)$ \\
Reasons for withdrawal & & $18(10.7)$ \\
Adverse event & $3(1.8)$ & $3(1.8)$ \\
Uncontrolled IOP & $3(1.8)$ & $2(1.2)$ \\
Protocol violation & $3(1.8)$ & $5(3.0)$ \\
Consent withdrawn & $0(0.0)$ & $0(0.0)$ \\
Lost to follow up & $1(0.6)$ & $1(0.6)$ \\
Other & $0(0.0)$ & \\
\hline IOP, intraocular pressure. &
\end{tabular}


favouring latanoprost/timolol treated patients (95\% CI -1.93 to $-0.72 ; \mathrm{p}<0.001)$. The mean (SD) per cent change in diurnal IOP was -35.7 (11.0) \% in patients treated with FC latanoprost/timolol and -30.7 (11.8) \% in those receiving UFC brimonidine/timolol $(\mathrm{p}<0.001)$. An exploratory, post hoc analysis in 21 patients receiving latanoprost and timolol at screening and who were randomised to FC latanoprost/timolol found an additional mean diurnal IOP reduction from screening to month 6 of 1.8 (SD 3.9) $\mathrm{mm} \mathrm{Hg}$ $(\mathrm{p}=0.003$; median reduction $=2.8 \mathrm{~mm} \mathrm{Hg}$; reduction range $=7.7 \mathrm{~mm} \mathrm{Hg}$ to $-12.0 \mathrm{~mm} \mathrm{Hg}$ ).

Reductions in mean IOP levels were greater at all measurement times in FC latanoprost/timolol treated patients (table 3). Adjusted mean IOP reductions in the FC latanoprost/timolol group were greater at 8:00AM $(-1.56 \mathrm{~mm} \mathrm{Hg} ; \quad 95 \%$ CI -2.24 to -0.89$), 12: 00$ noon $(-1.06 \mathrm{~mm} \mathrm{Hg} ; 95 \% \mathrm{CI}-1.70$ to -0.42$)$ and 4:00 PM $(-1.37 \mathrm{~mm} \mathrm{Hg}$; $95 \% \mathrm{CI}-2.02$ to -0.71$)(\mathrm{p} \leqslant 0.001$ for each comparison).

At month 6, 20.3\% of patients in the FC latanoprost/timolol group had an IOP $\leqslant 15 \mathrm{~mm} \mathrm{Hg}$ and $77.0 \%$ had an IOP $\leqslant 18 \mathrm{~mm} \mathrm{Hg}$ (fig 2); $13.6 \%$ and $55.0 \%$ of patients in the UFC brimonidine/timolol group had an IOP $\leqslant 15 \mathrm{~mm} \mathrm{Hg}$ and $\leqslant 18 \mathrm{~mm} \mathrm{Hg}$, respectively $(\mathrm{p}=0.1387$ and $\mathrm{p}<0.000 \mathrm{l}$ for differences between groups, respectively). Also after 6 months, $94.5 \%$ of the latanoprost/timolol group and $81.5 \%$ of the brimonidine/timolol group achieved $\geqslant 20 \%$ reductions in diurnal IOPs, and $74.8 \%$ of latanoprost/timolol treated patients and $58.6 \%$ of brimonidine/timolol treated patients achieved $\geqslant 30 \%$ reductions in diurnal IOP levels $(p=0.0003$ and $p=0.0022$ for differences between groups, respectively).

\section{Safety results}

Fewer patients receiving FC latanoprost/timolol reported an adverse event or a medication related adverse event (table 4). Drug related treatment emergent adverse events resulted in withdrawal from study medication in $1 / 165(0.6 \%)$ patients receiving FC latanoprost/timolol and in 19/167 (11.4\%) of those treated with UFC brimonidine/timolol. Five serious adverse events occurred, including one death in the brimonidine/timolol group and one case each of myocardial infarction, femoral arterial stenosis, haemorrhagic stroke, and myocarditis (not otherwise specified) in patients treated with latanoprost/timolol; no such event was considered related to study medication.

Fewer patients in the latanoprost/timolol group experienced an ocular adverse event (table 4). Hyperaemia occurred in no patient treated with FC latanoprost/timolol and in one patient treated with UFC brimonidine/timolol whereas red eye(s) was reported in one and three patients, respectively;

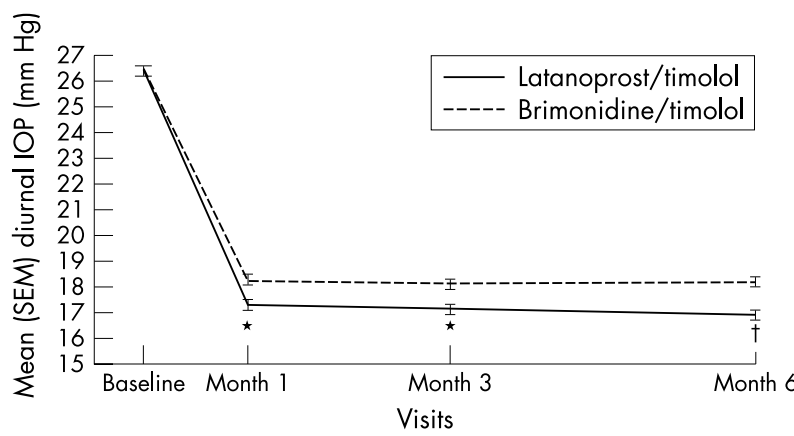

Figure 1 Unadjusted mean diurnal intraocular pressure (IOP) levels by treatment and visit intent to treat population. ${ }^{*} p=0.001,+p<0.001$, $t$ tests for difference between groups in mean IOP changes from baseline. SEM, standard error of the mean. one additional patient in the FC latanoprost/timolol group experienced painful red eyes. Treatment emergent allergic adverse events were noted in 4/165 (2.4\%) patients receiving FC latanoprost/timolol and in 13/167 (7.8\%) patients treated with UFC combination brimonidine/timolol.

At months 1, 3, and 6, new or worsening conditions found through lid/slit lamp examination were recorded in eight, 11, and five patients receiving FC latanoprost/timolol and in eight, nine, and 13 treated with UFC brimonidine/timolol, respectively. At each visit, improvement occurred in one patient in each treatment group. New or worsening conditions identified through ophthalmoscopy at months 3 and 6 were seen in zero and three patients treated with latanoprost/ timolol and in three and zero of those receiving brimonidine/ timolol, respectively; one patient receiving latanoprost/timolol had an improved condition at month 6 . In both groups, no notable results were seen with regard to cup:disc ratios, and vital signs were stable.

\section{DISCUSSION}

These results show that once daily administration of FC latanoprost/timolol is more effective than twice daily administration of UFC brimonidine/timolol in reducing IOP in patients with open angle glaucoma or ocular hypertension. This study was powered to detect a treatment difference of $1.5 \mathrm{~mm} \mathrm{Hg}$ in the change from IOP from baseline to month 6 using a $t$ test; the actual difference of $1.33 \mathrm{~mm} \mathrm{Hg}$ found using the more powerful ANCOVA was highly significant $(p<0.001)$, supporting the conclusion that once daily FC latanoprost/timolol more effectively reduces IOP levels than twice daily UFC brimonidine/timolol.

The efficacy and safety of brimonidine and latanoprost as adjunctive treatments to timolol have been compared in one short term, prospective trial. ${ }^{22}$ The authors focused on IOP reduction from baseline at peak drug effect and concluded that the two drugs were comparably effective. Although the authors' narrowly worded conclusion reflects their findings, the study's methodological shortcomings do not resolve the issue of the relative effectiveness of brimonidine and timolol versus latanoprost and timolol as UFC treatments. For example, IOP assessments at times other than that of the peak effect of brimonidine are not reported, nor is it pointed out that the IOP reduction at 8:00AM produced by latanoprost was approximately $65 \%$ to $85 \%$ greater than that produced by brimonidine. ${ }^{23}$ The authors note that glaucoma is a 24 hour disease and that it is important to evaluate the efficacy of drug therapy over the entire day and not just at the time of peak drug effect. However, they do not present brimonidine trough data, claiming a comparison with latanoprost's trough effect is problematic as the latter occurs at 8:00PM-a time when data are difficult to collect. Nevertheless, brimonidine's trough effect might usefully have been compared with latanoprost's effect at any time point, as studies ${ }^{24-27}$ have shown no consistently measurable peak or trough during a 24 hour period with once daily latanoprost administration.

Prospective, double masked trials have shown that once daily latanoprost is superior to twice daily brimonidine when used as monotherapy, ${ }^{28}$ and one retrospective study has found latanoprost to be superior to brimonidine when used adjunctively to timolol. ${ }^{30}$ Although no direct comparative studies were included in a meta-analysis of nine clinical trials of up to 6 months' duration, latanoprost reduced IOP levels more effectively than brimonidine $(p=0.045)$, a particularly salient result as all included brimonidine trials measured IOP at time of peak drug effect, whereas three of the six latanoprost studies used diurnal IOP measurements. ${ }^{31}$

Heterogeneity among glaucoma and ocular hypertension patients makes it difficult to establish optimal target IOP 


\begin{tabular}{|c|c|c|c|c|}
\hline & \multicolumn{2}{|l|}{ IOP } & \multicolumn{2}{|c|}{ IOP reduction: baseline to month 6} \\
\hline & $\begin{array}{l}\text { Fixed combination } \\
\text { latanoprost/timolol } \\
(n=163)\end{array}$ & $\begin{array}{l}\text { Unfixed combination } \\
\text { brimonidine/timolol } \\
(n=162)\end{array}$ & $\begin{array}{l}\text { Fixed combination } \\
\text { latanoprost/timolol } \\
(n=163)\end{array}$ & $\begin{array}{l}\text { Unfixed combination } \\
\text { brimonidine/timolol } \\
\text { ( } n=162 \text { ) }\end{array}$ \\
\hline \multicolumn{5}{|l|}{ Baseline } \\
\hline 8:00AM & $27.4(2.6)$ & $27.3(2.4)$ & & \\
\hline 12 noon & $26.3(3.1)$ & $26.3(3.2)$ & & \\
\hline 4:00PM & $25.5(3.4)$ & 25.8 (3.4) & & \\
\hline Diurnal & $26.4(2.7)$ & $26.5(2.8)$ & & \\
\hline \multicolumn{5}{|l|}{ Month 6} \\
\hline 8:00AM & $17.6(3.1)$ & $19.1(3.5)$ & $9.9(3.6)^{*}$ & $8.2(3.2)$ \\
\hline 2 noon & $16.8(2.9)$ & $17.8(3.2)$ & $9.5(3.9) \dagger$ & $8.4(3.9)$ \\
\hline 4:00PM & $16.3(3.0)$ & $17.7(3.2)$ & $9.2(3.9) \dagger$ & $8.1(4.2)$ \\
\hline Diurnal & $16.9(2.8)$ & $18.2(3.1)$ & $9.5(3.4)^{*}$ & $8.2(3.4)$ \\
\hline
\end{tabular}

level reductions. Findings of the Early Manifest Glaucoma Trial, though, have shown that the magnitude of IOP reduction is a major factor influencing disease progression. ${ }^{32}$ Progression risk was estimated to decrease by approximately $10 \%$ with each millimetre of mercury of IOP reduction. An IOP reduction of $30 \%$ has been shown to slow the rate of visual field progression among normotensive glaucoma patients, ${ }^{33}$ and it has been confirmed in ocular hypertension that even a more modest $20 \%$ reduction is an acceptable response to treatment. ${ }^{34}$ In the present study, $74.8 \%$ of those treated with latanoprost/timolol versus $58.6 \%$ of the brimonidine/timolol treated patients achieved $\geqslant 30 \%$ IOP reductions after 6 months, a magnitude likely to be clinically beneficial. Some clinicians prefer to set a target IOP level for their patients, and IOPs $\leqslant 18 \mathrm{~mm} \mathrm{Hg}$ have been associated with slowed disease progression in patients with ocular hypertension and glaucoma. ${ }^{35}$ Herein, more patients in the FC latanoprost/timolol group achieved IOP levels of $\leqslant 18 \mathrm{~mm} \mathrm{Hg}$ compared with the UFC brimonidine/timolol group $(77.0 \% \vee 55.0 \%$, respectively).

Fixed combination latanoprost/timolol more effectively lowered IOP levels at all measurement times. Such consistency is important because IOP fluctuations throughout the day are a significant risk factor for disease progression. ${ }^{37}$ Clinicians also should be aware that the use of oral nonsteroidal anti-inflammatory drugs with cyclooxygenase inhibitor activity-commonplace in the population most susceptible to glaucoma-may be associated with loss of otherwise consistent and reliably sustained IOP reducing efficacy with brimonidine. ${ }^{38}$

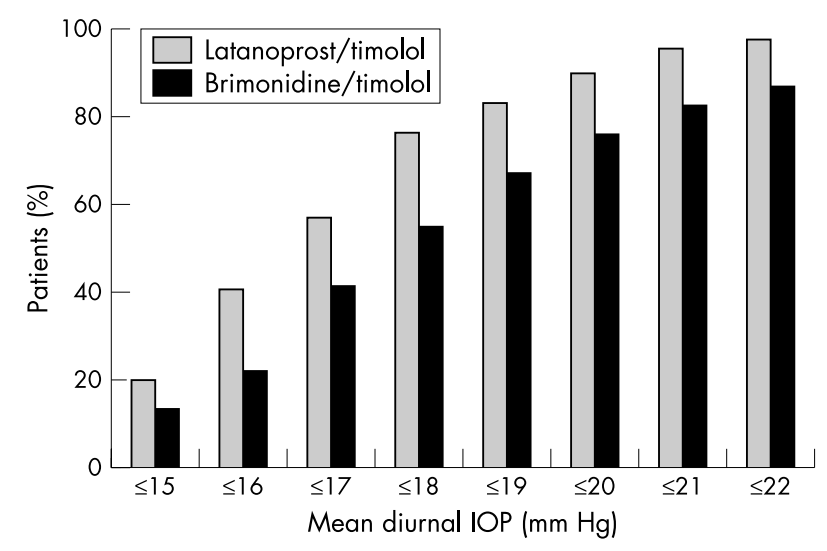

Figure 2 Percentages of patients reaching specified intraocular pressure (IOP) levels (intent to treat population).
In an exploratory, post hoc analysis, an additional mean diurnal IOP reduction of nearly $2 \mathrm{~mm} \mathrm{Hg}$ was observed in patients receiving maintenance therapy with UFC latanoprost/timolol at screening and who were randomised to receive FC latanoprost/timolol during the trial. Previous studies have shown that FC latanoprost/timolol provides greater IOP reducing efficacy than either of its individual components, with no substantial increase in adverse events. ${ }^{5}{ }^{14} 15$ The additional reduction found in this study may have been due partly to improved compliance reflecting the change from thrice to once daily instillation of drug. Simpler treatment schedules have been associated with enhanced patient compliance ${ }^{12}{ }^{13}$ whereas dosing with multiple drugs and having complex treatment schedules negatively affects patient quality of life. ${ }^{39}$

Finally, although both drugs generally were safe and well tolerated, medication related adverse events were reported more than twice as frequently in the UFC brimonidine/ timolol group and substantially more patients ( $11.4 \% v 0.6 \%$ receiving FC latanoprost/timolol) in that treatment group withdrew from the study because of a study medication related adverse event. Hyperaemia was not seen in the FC latanoprost/timolol group, although this side effect occurs in some patients treated with prostaglandin analogues. Allergic adverse events were noted in $7.8 \%$ of those treated with UFC brimonidine/timolol, a rate similar to that associated with the use of brimonidine monotherapy. ${ }^{28}$

\section{CONCLUSION}

The FC of latanoprost and timolol administered once daily is both more effective and better tolerated than twice daily dosing with the UFC of brimonidine and timolol.

Table 4 Adverse event summary

\begin{tabular}{|c|c|c|c|c|}
\hline & \multicolumn{2}{|c|}{$\begin{array}{l}\text { Fixed combination } \\
\text { latanoprost/timolol } \\
(n=165)\end{array}$} & \multicolumn{2}{|c|}{$\begin{array}{l}\text { Unfixed combination } \\
\text { brimonidine/timolol } \\
\text { ( } n=167 \text { ) }\end{array}$} \\
\hline & $\mathbf{n}$ & $\%$ & $\mathbf{n}$ & $\%$ \\
\hline No adverse event & 126 & 76.4 & 126 & 75.5 \\
\hline At least one adverse event & 39 & 23.6 & 41 & 24.6 \\
\hline Ocular adverse event(s) & 29 & 17.6 & 34 & 20.4 \\
\hline Systemic adverse event(s) & 14 & 8.5 & 13 & 7.8 \\
\hline $\begin{array}{l}\text { Adverse event(s) related to } \\
\text { study medication }\end{array}$ & 12 & 7.3 & 31 & 18.6 \\
\hline $\begin{array}{l}\text { Discontinuation due to } \\
\text { adverse event(s) }\end{array}$ & 3 & 1.8 & 18 & 10.8 \\
\hline Serious adverse event(s) & 4 & 2.4 & 1 & 0.6 \\
\hline
\end{tabular}




\section{Authors' affiliations}

J García-Sánchez, Hospital Clínico San Carlos, Madrid, Spain J-F Rouland, Hôpital Huriez, Lille, France

D Spiegel, Klinikum der Universitat Regensburg, Regensburg, Germany

B Paiic, Klinik Pallas, Olten, Switzerland

I Cunliffe, Solihull Hospital, Solihull, UK

C Traverso, Clinica Oculistica, DiNOG Università di Genova, Genova, Italy

J Landry, Pharmacia Corporation, Markham, Ontario, Canada

Correspondence to: Professor J García-Sánchez, Hospital Clínico San Carlos, Servicio de Oftalmología, Pabellón, 8, 5a Planta, Ciudad Universitaria s/n, 28040 Madrid, Spain; iiorcdir@med.ucm.es

Accepted for publication 3 November 2003

\section{MEMBERS OF THE EUROPEAN XALACOM STUDY GROUP}

France: Farida Bouzegaou, Hôpital Saint-Antoine, Paris Phillipe Denis, Hôpital Edouard Herriot, Lyon; JeanFrançois Huet, Centre Hospitalier André Mignot, Le Chesnay; Stéphane Jaulerry, Hôpital de Tarbes, Tarbes; Jean-Pierre Pisella, Hôpital Ambroise Paré Service Ophtalmologie, Boulogne; Wilford Williamson, Centre Hospitalier de Pau, Pau.

Germany: Kurt-Georg Berger, Private Practice, Friedrichsdorf; Heinrich Deuker, Private Practice, Darmstadt; Norbert Freiburg, Private Practice, Plettenberg; Thomas Hamacher, Private Practice, Starnberg; Maria-Luise Scherzer, Private Practice, Regenstauf; Ernst Schmack, Private Practice, Iserlohn; Dietmar Schnober, Private Practice, Werdohl; Reinhard Terlinde, Private Practice, Coesfeld.

Italy: Massimo G. Bucci, Cattedra di Oftalmología, Rome; E Gandolfo, U.O. di Oculistica, Brescia; Alfredo Reibaldi, Sezione di Oftalmología, Catania.

Spain: Alfonso Arias, Fundación Hospital Alcorcón, Alcorcón; Josep Callizo, Hospital Joan XIII, Tarragona; Francisco Honrubia, Hospital Miguel Servet, Zaragoza; Soledad Jiménez, Hospital Puerta del Mar, Cádiz; Manuel Sánchez-Salorio, Instituto Gallego de Oftalmología, Santiago de Compostela; Pedro Tañá, Hospital General de Alicante, Alicante; Miguel Teus, Hospital Príncipe de Asturias, Alcalá de Henares.

Switzerland: Jean-Jacques Tritten, Hôpital Communal Ophthalmologie, La Chaux-de-Fonds NE; E Ravinet, Hôpital Ophtalmique, Lausanne.

United Kingdom: C J MacEwen, Ninewells Hospital, Dundee; Donald Montgomery, Glasgow Royal Infirmary, Glasgow; R Sanders, Queen Margaret Hospital, Dunfermline; Stephen Vernon, Queen's Medical Centre, Nottingham.

\section{REFERENCES}

1 American Academy of Ophthalmology. Preferred practice pattern: primary open-angle glaucoma. San Francisco, CA: American Academy of Ophthalmology, 2000

2 European Glaucoma Society Committee for Education and Research. Terminology and Guidelines for Glaucoma. Savona, Italy: DOGMA S.r.I. 1998.

3 O'Connor DJ, Martone JF, Mead AM. Additive intraocular pressure lowering effect of various medications with latanoprost. Am J Ophthalmol 2002; 133:836-7

4 Hartenbaum D. The efficacy of dorzolamide, a topical carbonic anhydrase inhibitor, in combination with timolol in the treatment of patients with openangle glaucoma and ocular hypertension. Clin Ther 1996;18:460-5.

5 Diestelhorst M. Almegård B. Comparison of two fixed combinations of latanoprost and timolol in open-angle glaucoma. Graefe's Arch Clin Exp Ophthalmol 1998:236:577-81.

6 García-Sánchez J, and the Spanish Latanoprost Study Group. Efficacy and side effects of latanoprost monotherapy compared to adding dorzolamide to timolol in patients with glaucoma and ocular hypertension-a three-month randomised study. Eur J Ophthalmol 2000;10:198-204.

7 Toris CB, Camras CB, Yablonski ME. Effects of PhXA41, a new prostaglandin $\mathrm{F}_{2 \alpha}$ analog, on aqueous humor dynamics in human eyes. Ophthalmology 1993; 100:1297-304
8 Ziai N, Dolan JW, Kacere RD, et al. The effects on aqueous dynamics of $\mathrm{PhXA} 41$, a new prostaglandin $\mathrm{F} 2{ }_{\alpha}$ analogue, after topical application in normal and ocular hypertensive human eyes. Arch Ophthalmol 1993;111:1351-8.

9 Coakes RL, Brubaker RF. The mechanism of timolol in lowering intraocular pressure. In the normal eye. Arch Ophthalmol 1978;96:2045-8.

10 Rulo AH, Greve EL, Hoyng PF. Additive effect of latanoprost, a prostaglandin $\mathrm{F}_{2 \alpha}$ analogue, and timolol in patients with elevated intraocular pressure. Br J Ophthalmol 1994:78:899-902

11 Bucci MG, and the Italian Latanoprost Study Group. Intraocular pressure-lowering effects of latanoprost monotherapy versus latanoprost or pilocarpine in combination with timolol: a randomized, observer-masked multicenter study in patients with open-angle glaucoma. J Glaucoma 1999;8:24-30

12 Weinreb RN. Compliance with medical treatment of glaucoma. J Glaucoma 1992;1:134-6

13 Patel SC, Spaeth GL. Compliance in patients prescribed eyedrops for glaucoma. Ophthalmic Surg 1995;26:233-6.

14 Higginbotham EJ, Feldman R, Stiles $M$, et al. Latanoprost and timolol combination therapy vs monotherapy: one-year randomized trial. Arch Ophthalmol, 2002;120:915-22.

15 Pfeiffer N. A comparison of the fixed combination of latanoprost and timolol with its individual components. Graefe's Arch Clin Exp Ophthalmol 2002;240:893-9.

16 Toris $C B$, Gleason $M L$, Camras CB, et al. Effects of brimonidine on aqueous humor dynamics in human eyes. Arch Ophthalmol 1995:113:1514-917.

17 Toris CB, Camras CB, Yablonski ME. Acute versus chronic effects of brimonidine on aqueous humour dynamics in ocular hypertensive patients. Am J Ophthalmol 1999;128:8-14

18 Schuman JS, Horwitz B, Choplin NT, et al. A 1-year study of brimonidine twice daily in glaucoma and ocular hypertension. Arch Ophthalmol 1997; 115:847-52

19 Schuman JS. Clinical experience with brimonidine $0.2 \%$ and timolol $0.5 \%$ in glaucoma and ocular hypertension. Surv Ophthalmol 1996;41(Suppl 1): S27-S37.

20 LeBlanc RP, for the Brimonidine Study Group 2. Twelve-month results of an ongoing randomized trial comparing brimonidine tartrate $0.2 \%$ and timolol $0.5 \%$ given twice daily in patients with glaucoma or ocular hypertension. Ophthalmology 1998;105:1960-7.

21 Alphagan [package insert]. Irvine, CA: Allergan, Inc, 2002.

22 Simmons ST, Earl ML, for the Alphagan/Xalatan Study Group. Three-month comparison of brimonidine and latanoprost as adjunctive therapy in glaucoma and ocular hypertension patients uncontrolled on $\beta$-blockers: tolerance and peak intraocular pressure lowering. Ophthalmology 2002; 109:307-15

23 Camras CB. Brimonidine and latanoprost as adjunctive therapy Ophthalmology 2003;110:6-8

24 Camras CB, and the United States Latanoprost Study Group. Comparison of latanoprost and timolol inpatients with ocular hypertension and glaucoma. A six-month, multicenter trial in the United States. Ophthalmology $1996 ; 103: 138-47$

25 Camras CB, Wax MB, Ritch R, et al. Latanoprost treatment for glaucoma: effects of treating for 1 year and of switching from timolol. Am J Ophthalmol 1998;126:390-9.

26 Mishima HK, Masuda K, Kitazawa Y, et al. A comparison of latanoprost and timolol in primary open-angle glaucoma and ocular hypertension. A 12-week study. Arch Ophthalmol 1996;114:929-32.

27 Konstas AGP, Maltezos A, Gandi S, et al. Comparison of 24-hour intraocular pressure reduction with two dosing regimens of latanoprost and timolol maleate in patients with primary open-angle glaucoma. Am J Ophthalmol 1999; 128:15-20.

28 Kampik A, Arias-Puente A, O'Brart DPS, et al. Intraocular pressure-lowering effects of latanoprost and brimonidine therapy in patients with open-angle glaucoma or ocular hypertension: a randomized observer-masked multicenter study. J Glaucoma 2002;11:90-6.

29 Stewart WC, Day DG, Stewart JA, et al. The efficacy and safety of latanoprost $0.005 \%$ once daily versus brimonidine $0.2 \%$ twice daily in open-angle glaucoma or ocular hypertension. Am J Ophthalmol 2001;131:631-5.

30 Stewart WC, Sharpe ED, Day DG, et al. Comparison of the efficacy and safety of latanoprost $0.005 \%$ compared to brimonidine $0.2 \%$ or dorzolamide $2 \%$ when added to a topical beta-adrenergic blocker in patients with primary open-angle glaucoma or ocular hypertension. J Ocul Pharmacol Ther 2000;16:251-9.

31 Einarson TR, Kulin NA, Tingey D, et al. Meta-analysis of the effect of latanoprost and brimonidine on intraocular pressure in the treatment of glaucoma. Clin Ther 2000;22:1502-15.

32 Leske MC, Heiil A, Hussein M, et al. Factors for glaucoma progression and the effect of treatment: The Early Manifest Glaucoma Trial. Arch Ophthalmol 2003;121:48-56

33 Collaborative Normal Tension Glaucoma Study Group. Comparison of glaucomatous progression between untreated patients with normal-tension glaucoma and patients with therapeutically reduced intraocular pressures. Am J Ophthalmol 1998;126:487-97.

34 Kass MA, Hever DK, Higginbotham EJ, et al. The Ocular Hypertension Treatment Study: a randomized trial determines that topical ocular hypotensive medication delays or prevents the onset of primary open-angle glaucoma. Arch Ophthalmol 2002;120:701-13.

35 Mao LK, Stewart WC, Shields MB. Correlation between intraocular pressure control and progressive glaucomatous damage in primary open-angle glaucoma. Am J Ophthalmol 1991;111:51-5. 
36 AGIS Investigators. Advanced Glaucoma Intervention Study (AGIS): 7. The relationship between control of intraocular pressure and visual field deterioration. Am J Ophthalmol 2000;130:429-40.

37 Asrani SA, Zeimer R, Wilensky J, et al. Large diurnal fluctuations in intraocular pressure are an independent risk factor in patients with glaucoma. J Glaucoma 2000;9:134-42.
38 Sponsel WE, Paris G, Trigo Y, et al. Latanoprost and brimonidine: therapeutic and physiologic assessment before and after oral nonsteroidal anti-inflammatory therapy. Am J Ophthalmol 2002;133:11-18.

39 Perfetti S, Massagrandi S, Pagliani F. Glaucoma and quality of life. Acto Ophthalmol Scand 1998;227:52.

\section{$\mathrm{ECHO}$}

\section{Screening elderly people for poor sight is wasted effort}

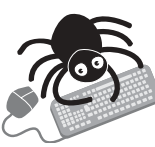

Please visit the British Journal of

Ophthalmology website [www. bjophthalmol. com] for a link to the full text of this article. randomised trial of screening for poor eyesight in elderly people in UK general practice
has concluded that universal screening is a waste of effort, the benefits are too limited.

The risk ratio for visual acuity $<6 / 18$ in either eye three to five years after screening was 1.07 for universal screening as against targeted screening in elderly subjects randomly drawn from two arms of an ongoing MRC trial assessing a range of health criteria. Both groups were comparable in their baseline values, but too few benefited from referral to an optician or ophthalmologist to lower the prevalence of impaired sight.

Twenty practices were randomly chosen from 106 general practices in the MRC trial and their patients assigned to complete a short assessment followed by detailed nurse assessment including a visual acuity test (universal screening) or a brief assessment and questions about problems with seeing and detailed assessment and acuity test only if the problems fell within a specified scope or severity (targeted screening). Almost 4400 patients aged 75 years or over took part.

Older people can easily lose their independence through poor vision and are then more susceptible to falls, lower quality of life, and depression. Current yearly vision screening is to become a single assessment for all patients of 75 or over, as proposed in the national service framework for older people. There is no evidence that screening is effective according to five earlier trials based on self reported visual acuity and, now, this superior study.

A British Medical Journal 2003;327:1027-1030.

\section{Video reports}

To view the video reports in full visit our website www. bjophthalmol.com and click on the link to video reports.

- Penetrating ocular injury due to a fish hook: surgical removal. SD M Chen, D Chiu, C K Patel

- Retinal ganglion cell axon response to guidance molecules. S F Oster, D W Sretavan

- Marin-Amat syndrome. A Jogiya, C Sandy

- Excision of subcutaneous dirofilariasis of the eyelid D Mallick, T P Ittyerah

- Thixotropy: a novel explanation for the cause of lagophthalmos after peripheral facial nerve palsy. M Aramideh, J H T M Koelman, P P Devriese, F VanderWerf, J D Speelman

- Surgical revision of leaking filtering blebs with an autologous conjunctival graft. $K$ Taherian, A Azuara-Blanco

- Dipetalonema reconditum in the human eye. T Huynh, J Thean, R Maini

- Evaluation of leucocyte dynamics in mouse retinal circulation with scanning laser ophthalmoscopy. H Xu, A Manivannan, G Daniels, J Liversidge, P F Sharp, J V Forrester, I J Crane

- An intraocular steroid delivery system for cataract surgery. D F Chang

- Pearls for implanting the Staar toric IOL. D F Chang

- Capsule staining and mature cataracts: a comparison of indocyanine green and trypan blue dyes. D F Chang

- Endoscopy to aid anterior segment surgery. J E Moore, A Sharma

- Reconstruction of the Ocular Surface in LOGIC Syndrome. J E Moore, V Kumar, $J$ R Ainsworth, S Shah

- A suture technique to manage a case of severe early flap displacement after laser in situ keratomileusis. L Spadea, P Pantaleoni, G Bianco 